Original Research Article

\title{
Treatment of acute ischemic stroke with recombinant tissue plasminogen activator: practice pattern among neurologists and physicians
}

\author{
Yaseen Mohammed, Sarala N.*, Asha B., Bhuvana K.
}

Department of Pharmacology, Sri Devaraj Urs Medical College, Sri Devaraj Urs University of Higher Education and Research, Tamaka, Kolar, Karnataka, India

Received: 07 November 2016 Accepted: 09 December 2016

*Correspondence to:

Dr. Sarala N.,

Email: n_sarala@rediffmail.com

Copyright: (C) the author(s), publisher and licensee Medip Academy. This is an openaccess article distributed under the terms of the Creative Commons Attribution NonCommercial License, which permits unrestricted noncommercial use, distribution, and reproduction in any medium, provided the original work is properly cited.

\begin{abstract}
Background: Stroke is an abrupt onset of a neurologic deficit due to a focal vascular disease. Treatment guidelines for acute ischemic stroke (AIS) within 4.5 hours of onset are thrombolysis with recombinant tissue plasminogen activator (rtPA). To determine the practice pattern of rtPA in the treatment of acute ischemic stroke among consultants using a questionnaire.

Methods: A questionnaire based study was carried out from May to September 2015. Neurologists and physicians from Kolar and Bengaluru were given a questionnaire comprising of 21 questions, regarding the treatment of AIS with rtPA. The data was analyzed using descriptive statistics.

Results: A total of $76.9 \%$ responded to the questionnaire, of which 18 were neurologists and 82 were physicians. An average of 4-5 AIS patients per month were seen by the doctors. Majority $(72 \%)$ did not use rtPA, due to delay in patient reaching hospital or non-affordability of the drug. The consultants $(66 \%)$ opined that rtPA was the best if patient arrived within 4.5 hours of onset of AIS. Only $34 \%$ consultants knew the correct score of AIS for administration of rtPA. The usage of penumbral imaging before thrombolysis was agreed by neurologists $(58 \%)$ and physicians $(34 \%)$. The IV+IA rtPA thrombolytic therapy produced highest rate of recanalization as expressed by neurologists $(84 \%)$ and physicians $(56 \%)$. Majority $(80 \%)$ felt that use of rtPA beyond 4-6 hours had no beneficial effect. The adverse effects encountered were hypotension and bleeding.

Conclusions: Neurologists and physicians opined that treatment with rtPA was effective in patients of AIS within 4.5 hours of onset, but the limitations were late arrival of patient to hospital and drug cost.
\end{abstract}

Keywords: Acute ischemic stroke, Neurologists, Practice patterns, Physicians, Recombinant tissue plasminogen activator

\section{INTRODUCTION}

Stroke is a neurologic deficit of an abrupt onset due to a focal vascular disease. Neurologic symptoms will manifest within seconds due to lack of glycogen in neurons. Sudden occlusion of an intracranial vessel results in decreased blood flow to an area of the brain it supplies. Therefore ischemia can occur if the cerebral blood flow reduces to $<20 \mathrm{ml} / 100 \mathrm{~g}$ tissue per minute. ${ }^{1}$ Ischemia of neurons depletes glycogen which results in neurologic symptoms.

Treatment for acute ischemic stroke (AIS) within 4.5 hours of onset of stroke is thrombolysis, which refers to lysis of thrombus by stimulating fibrinolysis. Use of intravenous recombinant tissue plasminogen activator (rtPA) for AIS is found to be highly effective. ${ }^{2}$ The National Institute of Neurological Disorders and Stroke (NINDS) had conducted a study on efficacy of rtPA in the treatment of AIS, the results were found to be clinically and statistically significant. ${ }^{3}$ The use of intravenous rtPA for the treatment of AIS is approved by Food and Drug Administration (FDA) and Drug Controller General of India (DGCI). ${ }^{4}$

Alteplase, a rtPA when administered intravenously in AIS has shown improvement in mobility, self-care and quality of life post treatment in all age groups. ${ }^{5}$ The 
treatment for AIS has barriers like delayed arrival of patient, lack of awareness about thrombolysis for stroke among medical professionals, deficiency of centers offering thrombolysis and patient's affordabilty. ${ }^{6}$ The availability of rtPA in the hospital is one of the most important factor for the better effectiveness of thrombolysis during window period. ${ }^{3}$ Thrombolysis with rtPA is found to be cost-effective both in terms of inpatient hospitalization and the stroke care at home. ${ }^{4}$ Yet the usage of rtPA for AIS has been limited. Hence this study was carried out to determine the perception and practice patterns among neurologists and physicians in the treatment of acute ischemic stroke with rtPA using questionnaire.

\section{METHODS}

This was a cross-sectional, questionnaire based study carried out by the department of Pharmacology, attached to Sri Devaraj Urs Medical College, Tamaka, Kolar from May to September 2015. The study protocol was approved by Institutional Ethics Committee. Written informed consent was obtained from all the doctors who were willing to participate in the study. The questionnaire comprising of 21 questions, regarding the treatment of AIS with rtPA were distributed in person to the neurologists and physicians working in Kolar and Bengaluru and 20 minutes' time was given to complete the same. Questionnaire along with consent form was also mailed to the doctors and were requested to complete it and return. The questionnaire was divided into three parts. The first part included details of educational qualification and designation of respondents. The second part questions were designed to assess the clinical use of rtPA in AIS patients. The third part of the questionnaire was addressed to the use of rtPA in AIS patients with different co-morbidities. The data was analyzed using descriptive statistics.

\section{RESULTS}

A total of 130 questionnaires were distributed to doctors. One hundred filled questionnaires were received, therefore the response rate was $76.9 \%$, of which 18 were neurologists and 82 were physicians. An average of 4-5 AIS patients per month were seen by them, $72 \%$ consultants did not use rtPA for the treatment of these patients and the reasons expressed are represented in Figure 1.

Neurologist $(68 \%)$ and physicians $(32 \%)$ felt that rtPA was the best treatment option if the patient arrived within 4.5 hours of AIS onset. Only $34 \%$ of consultants knew the correct Number Needed to Treat (NNT) score of AIS for administration of $\mathrm{rtPA}$, of them $41 \%$ were neurologists and $27 \%$ were physicians. The clinical usage of penumbral imaging to select the patient before thrombolysis was agreed by neurologists (58\%) and physicians $(34 \%)$. The intravenous (IV) + intra-arterial (IA) rtPA thrombolytic therapy produced highest rate of recanalization in AIS as expressed by neurologists (84\%) and physicians $(56 \%)$.

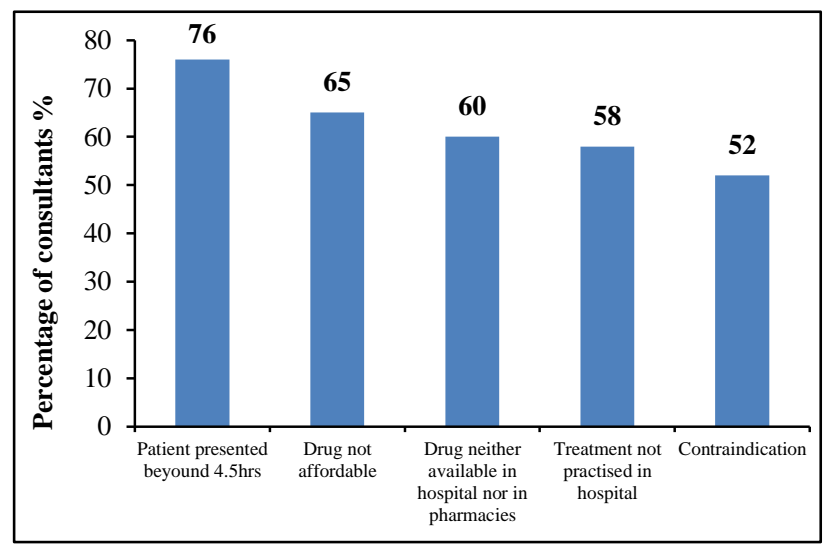

Figure 1: Reasons for not using rtPA.

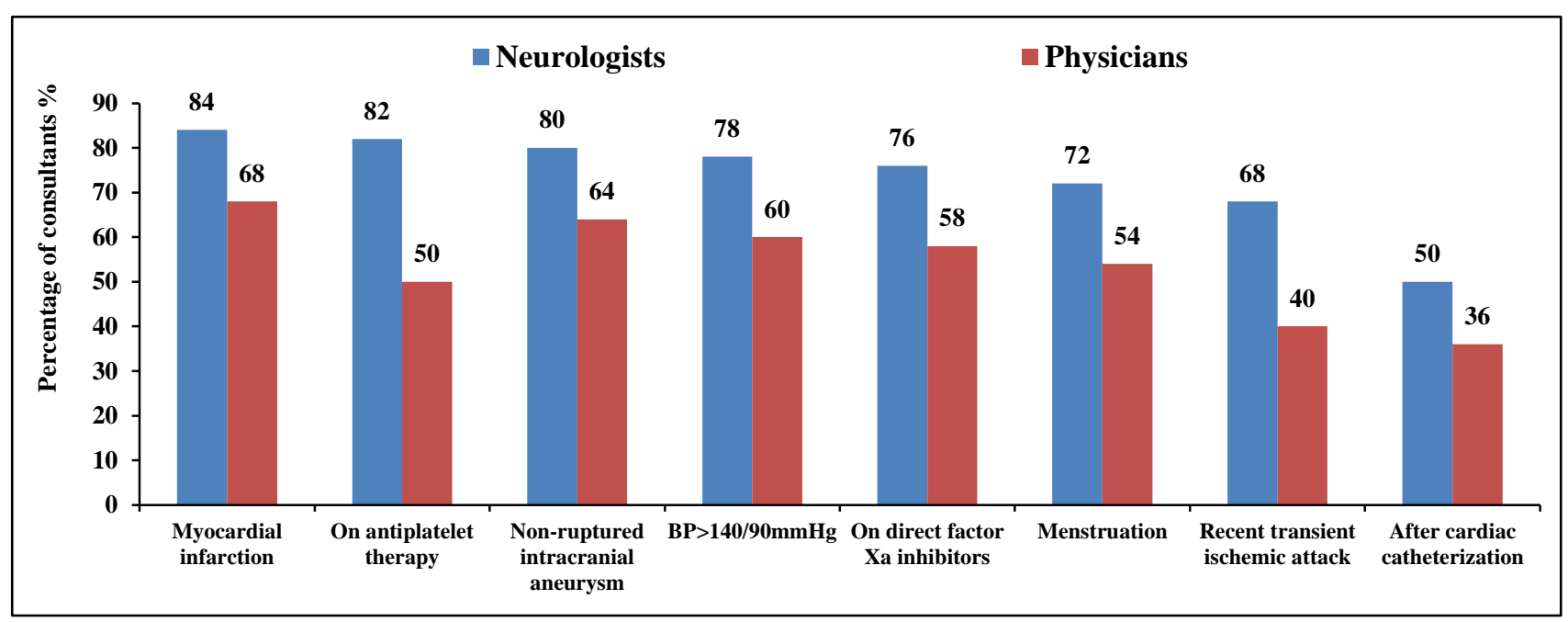

Figure 2: Use of rtPA in different co-morbid conditions. 
The consultants agreed that use of rtPA was safe in AIS when associated with different clinical situations as shown in Figure 2.

The risk of intracranial hemorrhage (ICH) in AIS patients treated with intravenous rtPA is less than one percent as expressed by neurologists (74\%) and physicians $(30 \%)$. Eighty percent of consultants felt that use of rtPA beyond 4-6 hours had no beneficial effect. The common adverse effect encountered by them was hypotension apart from bleeding.

\section{DISCUSSION}

Acute ischemic stroke is a medical emergency and early intervention can limit the extent of ischemic brain injury, prevent complications like brain edema, haemorrhagic transformation, facilitate early recovery and reduce morbidity and mortality. The effective management of AIS is either with rtPA or by mechanical thrombolectomy. The current treatment for AIS approved by U.S FDA is administration of alteplase within 3hours of onset of symptoms. ${ }^{7}$

Alteplase, tenecteplase and reteplase are fibrinolytics which convert fibrin bound plasminogen to plasmin resulting in lysis of the thrombus rapidly. ${ }^{8}$ In our study majority of consultants were not using alteplase and the reasons expressed were delay in patients reaching the hospital, patient could not afford the drug and medication not being accessible. A study revealed that only a small proportion of the patients with AIS are currently treated with rtPA like alteplase because of the narrow time window and contraindications for its use. ${ }^{9}$ The above mentioned factors can be overcome by educating people during health programmes to recognise early symptoms and signs of stroke, its complications and creating awareness among patients regarding the importance of timely administration of alteplase. Most of the consultants expressed that rtPA was the best option if patient reached the hospital within the window period. A recent study has revealed that half the dose of alteplase is as effective as full dose with significantly less bleeding, which will benefit the economically weaker section of patients. ${ }^{10}$

In this study the percentage of physicians who were aware about the choice of best treatment option for AIS and also the effectiveness of IV+IA thrombolytic therapy was less. To thrombolyse effectively all patients with AIS arriving at the hospital and to reduce morbidity and mortality following stroke, the physicians and neurologists should be aware about the use of rtPA during the window period. This can be achieved if the primary physicians who are the first contact to most of the patients in rural and urban areas are to be updated regarding initiating early intervention for AIS with alteplase and its advantages.
In this study it was observed that the percentage of the physicians using rtPA for AIS within the treatment window period was low. The awareness of thrombolytic therapy was high among the neurologists, which reflects that use of rtPA by specialists at higher centres is more common, but it is beyond the reach of patients to arrive at these centers on time. A study has shown that there is association between knowledge of contraindications and thrombolytic therapy, in our study there was awareness among the consultants in respect to co-morbid conditions. $^{4}$ The primary physicians should have knowledge regarding the safety of the fibrinolytics.

\section{CONCLUSION}

Neurologists and physicians opined that treatment with rtPA was effective in patients of AIS within 4.5 hours of onset, but the late arrival to hospital and drug cost were limitations for thrombolytic therapy. These observations direct us that the strategies should be developed to create awareness among the consultants about importance of window period for thrombolysis in their daily practice.

Funding: No funding sources

Conflict of interest: None declared

Ethical approval: The study was approved by the Institutional Ethics Committee

\section{REFERENCES}

1. Smith WS, English JD, Johnston SC. Cerebrovascular diseases. Longo DL, Fauci AS, Kasper DL, Hauser DL, Jameson JL, Loscalzo J. In Harrison's Principles of Internal Medicine. 19th ed., New York, McGraw Hill publication; 2012:32713291.

2. Schmidt HG. In Intravenous thrombolysis for acute ischemic stroke: from randomized clinical trials to daily practice. Netherlands. Maaike dirks publication. 2012:15-7.

3. Dirks M, Niessen LW, Koudstaal PJ, Franke CL, Oostenbrugge RJV, Dippel DWJ. Intravenous thrombolysis in acute ischaemic stroke: from trial exclusion criteria to clinical contraindications. An international Delphi study. J Neurol Neurosurg Psychiatry. 2007;78:685-9.

4. Edlow JA, Smith EE, Stead LG, Gronseth G, Messe SR, Jagoda AS et al. Clinical policy: use of intravenous tPA for the management of acute ischemic stroke in the emergency department. Ann Emerg Med. 2013;61:225-43.

5. Sandercock P, Wardlaw JM, Dennis M, Cohen G, Murray G, Innes $\mathrm{K}$ et al. Effect of thrombolysis with alteplase within 6 hour of acute ischemic stroke on long-term outcomes (the third International Stroke Trial [IST-3]): 18-month follow-up of a randomized controlled trial. Lancet neurology. 2013;12:768-76.

6. Aaron S, Alexander M, Maya T, Mathew V, Goyal M. Treatment of acute ischemic stroke: awareness 
among general practioners. Neurology India. 2010;58:441-2.

7. Goldstein LB. Modern medical management of acute ischemic stroke. MDCVJ. 2014;10(2):99-104.

8. Zehnder JL. Drugs used in disorders of coagulation. Katzung BG, Trevor AJ. In Basic and clinical pharmacology. $13^{\text {th }}$ ed. Newyork, McGraw Hill; 2015:879-880.

9. Dirks M, Niessen LW, Huijsman R, van Wijngaarden J, Minkman MMN, Franke C et al. Promoting acute thrombolysis for ischaemic stroke. Protocol for a cluster randomised controlled trial to assess the effect of implementation strategies on the rate and effects of thrombolysis for acute ischaemic stroke. International Journal of Stroke. 2007;2:151-9.

10. Pandian JD, Sudan P. Stroke epidemiology and stroke care services in India. $\mathbf{J}$ of Stroke. 2013;15:128-34.

Cite this article as: Mohammed Y, Sarala N, Asha $\mathrm{B}$, Bhuvana K. Treatment of acute ischemic stroke with recombinant tissue plasminogen activator: practice pattern among neurologists and physicians. Int J Basic Clin Pharmacol 2017;6:180-3. 